Article

\title{
Subordination and Superordination Properties for Certain Family of Analytic Functions Associated with Mittag-Leffler Function
}

\author{
Mansour F. Yassen ${ }^{1,2}$, Adel A. Attiya ${ }^{3,4}$ and Praveen Agarwal 5,6,7,8,* \\ 1 Department of Mathematics, College of Science and Humanities in Al-Aflaj, \\ Prince Sattam Bin Abdulaziz University, Al-Aflaj 11912, Saudi Arabia; mf.ali@psau.edu.sa \\ 2 Department of Mathematics, Faculty of Science, Damietta University, New Damietta 34517 Damietta, Egypt \\ 3 Department of Mathematics, College of Science, University of Ha'il, Ha'il 81451, Saudi Arabia; \\ aattiy@mans.edu.eg \\ 4 Department of Mathematics, Faculty of Science, University of Mansoura, Mansoura 35516, Egypt \\ 5 Department of Mathematics, Anand International College of Engineering, Jaipur 303012, India \\ 6 International Center for Basic and Applied Sciences, Jaipur 302029, India \\ 7 Institute of Mathematics and Mathematical Modeling, Almaty 050010, Kazakhstan \\ 8 Harish-Chandra Research Institute (HRI), Allahabad 211 019, India \\ * Correspondence: praveen.agarwal@anandice.ac.in
}

Received: 24 August 2020; Accepted: 9 October 2020; Published: 19 October 2020

check for updates

\begin{abstract}
We obtain new outcomes of analytic functions linked with operator $\mathcal{H}_{\alpha, \beta}^{\eta, k}(f)$ defined by Mittag-Leffler function. Moreover, new theorems of differential sandwich-type are obtained.
\end{abstract}

Keywords: differential subordinations; differential superordinations; subordinant; Mittag-Leffler function

\section{Basic Definitions and Preliminaries}

Let $\mathbb{A}$ define the class of analytic functions in the open unit disk $\mathbb{U}=\{z \in \mathbb{C}:|z|<1\}$ and let $\mathcal{H}[a, n]$ be the subclass of $\mathbb{A}$, which is

$$
f(z)=a+a_{n} z^{n}+a_{n+1} z^{n+1}+a_{n+2} z^{n+2}+a_{n+3} z^{n+3}+\ldots \quad(a \in \mathbb{C}),
$$

Furthermore, let $\mathcal{H}$ be the subclass of $\mathbb{A}$ of all the functions $f(z) \in \mathcal{H}$ normalized by

$$
f(z)=z+\sum_{n=2}^{\infty} a_{n} z^{n}
$$

Attiya [1] introduced and investigated the operator $\mathcal{H}_{\alpha, \beta}^{\eta, k}(f(z)): \mathcal{H} \rightarrow \mathcal{H}$, which $\mathcal{H}_{\alpha, \beta}^{\eta, k}(f(z))$ is defined by

$$
\mathcal{H}_{\alpha, \beta}^{\eta, k}(f(z))=Q_{\alpha, \beta}^{\eta, k}(z) * f(z), \quad(z \in \mathbb{U}),
$$

for $f(z) \in \mathcal{H}$ given by $(1)$, the symbol $*$ denotes the Hadamard product, and

$$
Q_{\alpha, \beta}^{\eta, k}(z)=\frac{\Gamma(\alpha+\beta)}{(\eta)_{k}}\left(E_{\alpha, \beta}^{\eta, k}(z)-\frac{1}{\Gamma(\beta)}\right), \quad(z \in \mathbb{U})
$$

Moreover, the function $E_{\alpha, \beta}^{\eta, k}(z)$ is called the general Mittag-Leffler function defined by

$$
E_{\alpha, \beta}^{\eta, k}(z)=\sum_{n=0}^{\infty} \frac{(\eta)_{n k} z^{n}}{\Gamma(\alpha n+\beta) n !}, \quad(\alpha, \beta, \eta \in \mathbb{C} ; \operatorname{Re}(\alpha)>\max \{0, \operatorname{Re}(k)-1\} ; \operatorname{Re}(k)>0)
$$


where

$$
(\eta)_{n}=\frac{\Gamma(\eta+n)}{\Gamma(\eta)}= \begin{cases}1, & n=0 \\ \eta(\eta+1)(\eta+2) \ldots(\eta+n-1), & n \in \mathbb{N}\end{cases}
$$

The function $E_{\alpha, \beta}^{\eta, k}(z)$ was investigated by Srivastava and Tomovski [2]. Many authors studied and investigated Mittag-Leffler function; for more details on Mittag-Leffler function and general Mittag-Leffler function see, e.g., [1,3-19].

Moreover, Attiya [1] deduced that $\mathcal{H}_{\alpha, \beta}^{\eta, k}(f(z))$ can be put in

$$
\mathcal{H}_{\alpha, \beta}^{\eta, k}(f(z))=z+\sum_{n=2}^{\infty} \frac{\Gamma(\eta+n k) \Gamma(\alpha+\beta)}{\Gamma(\eta+k) \Gamma(n \alpha+\beta)} a_{n} z^{n} \quad(z \in \mathbb{U}) .
$$

It follows from (2) that (see [1])

$$
k z\left(\mathcal{H}_{\alpha, \beta}^{\eta, k}(f(z))\right)^{\prime}=(\eta+k)\left(\mathcal{H}_{\alpha, \beta}^{\eta+1, k}(f(z))\right)-\eta\left(\mathcal{H}_{\alpha, \beta}^{\eta, k}(f(z))\right),
$$

and

$$
\alpha z\left(\mathcal{H}_{\alpha, \beta+1}^{\eta, k}(f(z))\right)^{\prime}=(\alpha+\beta)\left(\mathcal{H}_{\alpha, \beta}^{\eta, k}(f(z))\right)-\beta\left(\mathcal{H}_{\alpha, \beta+1}^{\eta, k}(f(z))\right) .
$$

It should be remarked that the operator $\mathcal{H}_{\alpha, \beta}^{\eta, k}(f(z))$ for some special cases of $\alpha, \beta, \eta$, and $k$ provides many special functions, e.g.,

$$
\begin{aligned}
& \mathcal{H}_{0, \beta}^{1,1}(f)(z)=f(z) . \\
& \mathcal{H}_{0,1}^{2,1}(f)(z)=\frac{1}{2}\left(f(z)+z f^{\prime}(z)\right) . \\
& \mathcal{H}_{0, \beta}^{0,1}(f)(z)=\int_{0} \frac{1}{t} f(t) d t . \\
& \mathcal{H}_{1,0}^{1,1}\left(\frac{z}{1-z}\right)=z e^{z} . \\
& \mathcal{H}_{1,1}^{1,1}\left(\frac{z}{1-z}\right)=e^{z}-1 . \\
& \mathcal{H}_{2,1}^{1,1}\left(\frac{z}{1-z}\right)=-2+\cosh (\sqrt{z}) .
\end{aligned}
$$

Definition 1. Let functions $f(z)$ and $g(z)$ be analytic in the open unit disk $\mathbb{U}$. Then $f(z)$ is subordinate to $g(z)$ if there exists a Schwarz function $\omega(z)$, analytic in $\mathbb{U}$ with $\omega(0)=0$ and $|\omega(z)|<1,(z \in \mathbb{U})$, such that $f(z)=g(\omega(z)),(z \in \mathbb{U})$, we denote this subordination by $f(z) \prec g(z)$. In particular, if $g(z)$ is univalent in $\mathbb{U}$, then subordination is equivalent to $f(z) \prec g(z) \Leftrightarrow f(0)=g(0)$ and $f(\mathbb{U}) \subset g(\mathbb{U})$.

Definition 2. If $\mathbb{Q}$ the set of all functions $q(z)$ that are analytic and univalent on $\overline{\mathbb{U}} \backslash E(q)$, where

$$
E(q)=\left\{\xi \in \partial \mathbb{U}: \lim _{z \rightarrow \xi} q(z)=\infty\right\},
$$

and $\min \left|q^{\prime}(\xi)\right|=\rho>0$ for $\xi \in \partial \mathbb{U} \backslash E(q)$. Further, let $\mathbb{Q}(a)=\{q(z) \in \mathbb{U}: q(0)=a\}$ and $\mathbb{Q}_{1}=\mathbb{Q}(1)$.

Definition 3. If $\psi: \mathbb{C}^{4} \times \mathbb{U} \longrightarrow \mathbb{C}$ and $h(z)$ be univalent in $\mathbb{U}$. If $p(z)$ is analytic in $\mathbb{U}$, and satisfies the third-order differential subordination

$$
\psi\left(p(z), z p^{\prime}(z), z^{2} p^{\prime \prime}(z), z^{3} p^{\prime \prime \prime}(z) ; z\right) \prec h(z),
$$

then $p(z)$ is called a solution of the differential subordination and $q(z)$ is called a dominant of the solutions of the differential subordination as well as a dominant if $p(z) \prec q(z)$ for all $p(z)$ satisfying (5). $\widetilde{q}(z)$ that satisfies $\widetilde{q}(z) \prec q(z)$ for all dominants of (5) is called the best dominant of (5). 
Definition 4. Let $\Omega \subseteq \mathbb{C}, q(z) \in \mathbb{Q}$ and $n \in \mathbb{N} \backslash\{1\}$. The class of admissible functions $\Psi_{n}[\Omega, q(z)]$ consists of those functions $\psi: \mathbb{C}^{4} \times \mathbb{U} \longrightarrow \mathbb{C}$ that satisfy the admissibility condition:

$$
\psi(r, s, t, u ; z) \notin \Omega,
$$

whenever

$$
r=q(\zeta), \quad s=\ell \zeta q^{\prime}(\zeta), \quad \operatorname{Re}\left(\frac{t}{s}+1\right) \geq \ell \quad \operatorname{Re}\left(\frac{\zeta q^{\prime \prime}(\zeta)}{q^{\prime}(\zeta)}+1\right)
$$

and

$$
\operatorname{Re}\left(\frac{u}{s}\right) \geq \ell^{2} \quad \operatorname{Re}\left(\frac{\zeta^{2} q^{\prime \prime \prime}(\zeta)}{q^{\prime}(\zeta)}\right)
$$

where $z \in \mathbb{U} ; \zeta \in \partial \mathbb{U} \backslash E(q)$ and $\ell \geq n$.

Analogous to the second order differential super-ordinations introduced by Miller and Mocanu [20], Tang et al. [21] defined the differential super-ordinations as follows:

Definition 5. Let $\psi: \mathbb{C}^{4} \times \mathbb{U} \longrightarrow \mathbb{C}$ and the function $h(z)$ be analytic in $\mathbb{U}$. If functions $p(z)$ and $\psi\left(p(z), z p^{\prime}(z), z^{2} p^{\prime \prime}(z), z^{3} p^{\prime \prime \prime}(z)\right)$ are univalent in $\mathbb{U}$, and satisfy the following third-order differential super-ordination

$$
h(z) \prec \psi\left(p(z), z p^{\prime}(z), z^{2} p^{\prime \prime}(z), z^{3} p^{\prime \prime \prime}(z)\right),
$$

then $p(z)$ is called a solution of the differential superordination and $q(z)$ is called a subordinant of the solutions of the differential super-ordinations as well as a subordinant if $p(z) \prec q(z)$ for all $p(z)$ satisfying Equation (6). A univalent subordinant $\widetilde{q}(z)$ that satisfies $\widetilde{q}(z) \prec q(z)$ for all super-ordinations of $(6)$ is the best superordinant.

Definition 6. Let $\Omega \subseteq \mathbb{C}, q(z) \in \mathcal{H}[a, n]$ with $n \in \mathbb{N} \backslash\{1\}$ and $q^{\prime}(z) \neq 0$. The class of admissible functions $\Psi_{n}^{\prime}[\Omega, q(z)]$ consists of those functions $\psi: \mathbb{C}^{4} \times \mathbb{U} \longrightarrow \mathbb{C}$ that satisfy the admissibility condition:

$$
\psi(r, s, t, u ; \zeta) \in \Omega
$$

whenever

$$
r=q(z), \quad s=\frac{z q^{\prime}(z)}{m}, \quad \operatorname{Re}\left(\frac{t}{s}+1\right) \leq \frac{1}{m} \quad \operatorname{Re}\left(\frac{z q^{\prime \prime}(z)}{q^{\prime}(z)}+1\right)
$$

and

$$
\operatorname{Re}\left(\frac{u}{s}\right) \leq \frac{1}{m^{2}} \quad \operatorname{Re}\left(\frac{z^{2} q^{\prime \prime \prime}(z)}{q^{\prime}(z)}\right)
$$

where $z \in \mathbb{U} ; \zeta \in \partial \mathbb{U}$ and $m \geq n \geq 2$.

Here, we use the following theorems given by Antonino and Miller [22]:

Theorem 1 ([22]). Let $p(z) \in \mathcal{H}[a, n]$ with $n \in \mathbb{N} \backslash\{1\}$. Also, let $q(z) \in \mathbb{Q}(a)$ and satisfy the following conditions:

$$
\begin{aligned}
& \operatorname{Re}\left(\frac{\zeta q^{\prime \prime}(\zeta)}{q^{\prime}(\zeta)}\right)>0, \quad\left|\frac{z p^{\prime}(z)}{q^{\prime}(\zeta)}\right| \leq \ell, \quad(z \in \mathbb{U} ; \zeta \in \partial \mathbb{U} \backslash E(q) ; \ell \geq n), \\
& \psi\left(p(z), z p^{\prime}(z), z^{2} p^{\prime \prime}(z), z^{3} p^{\prime \prime \prime}(z) ; z\right) \in \Omega,
\end{aligned}
$$

and, if $\Omega \subseteq \mathbb{C}, \psi \in \Psi_{n}[\Omega, q(z)]$, then $p(z) \prec q(z)$. 
Theorem 2. Let $q(z) \in \mathcal{H}[a, n]$ and $\psi \in \Psi_{n}^{\prime}[\Omega, q(z)]$. If $p(z) \in \mathbb{Q}(a)$ and $\psi\left(p(z), z p^{\prime}(z), z^{2} p^{\prime \prime}(z), z^{3} p^{\prime \prime \prime}(z) ; z\right)$ is univalent in $\mathbb{U}$ and

$$
\begin{aligned}
& \operatorname{Re}\left(\frac{z q^{\prime \prime}(z)}{q^{\prime}(z)}\right) \geq 0, \quad\left|\frac{\zeta p^{\prime}(\zeta)}{q^{\prime}(z)}\right| \leq m, \quad(z \in \mathbb{U} ; \zeta \in \partial \mathbb{U} ; m \geq n \geq 2) \\
& \Omega \subset\left\{\psi\left(p(z), z p^{\prime}(z), z^{2} p^{\prime \prime}(z), z^{3} p^{\prime \prime \prime}(z) ; z\right): z \in \mathbb{U}\right\}
\end{aligned}
$$

then $q(z) \prec p(z)$.

Here, we study a certain family of admissible functions by using the third-order differential subordination and superordination given by Antonino and Miller [22] and Tang et al. [21]—see also Attiya et al. [23] —we obtain new results of subordination and superordination properties of analytic functions linked with the operator $\mathcal{H}_{\alpha, \beta}^{\eta, k}(f)$.

\section{Main Results}

Definition 7. Let $\Omega \subseteq \mathbb{C}$ and $q(z) \in \mathbb{Q}$. The class of admissible functions $\Psi_{\Gamma}[\Omega, q(z)]$ consists of those functions $\phi: \mathbb{C}^{4} \times \mathbb{U} \longrightarrow \mathbb{C}$ that satisfy the admissibility condition

$$
\phi\left(a_{1}, a_{2}, a_{3}, a_{4} ; z\right) \notin \Omega,
$$

whenever

$$
\begin{gathered}
a_{1}=q(\zeta), \quad a_{2}=\frac{\ell k \zeta q^{\prime}(\zeta)+b q(\zeta)}{b}, \\
\operatorname{Re}\left(\frac{(b+1)\left(a_{3}-a_{1}\right)}{k\left(a_{2}-a_{1}\right)}-\frac{2 b+1}{k}\right) \geq \ell \operatorname{Re}\left(\frac{\zeta q^{\prime \prime}(\zeta)}{q^{\prime}(\zeta)}+1\right), \\
\operatorname{Re}\left(\frac{(b+1)(b+2)\left(a_{4}-a_{1}\right)-3(b+1)(b+k+1)\left(a_{3}-a_{1}\right)}{k^{2}\left(a_{2}-a_{1}\right)}+\frac{3 b(b+1)+1}{k^{2}}\right. \\
\left.+\frac{6 b+3}{k}+2\right) \geq \ell^{2} \operatorname{Re}\left(\frac{\zeta^{2} q^{\prime \prime \prime}(\zeta)}{q^{\prime}(\zeta)}\right),
\end{gathered}
$$

where $z \in \mathbb{U} ; \zeta \in \partial \mathbb{U} \backslash E(q), \ell \in \mathbb{N} \backslash\{1\}$ and $b=\eta+k$.

Theorem 3. Let $\phi \in \Psi_{\Gamma}[\Omega, q(z)]$. If $f(z) \in \mathcal{H}$ and $q(z) \in \mathbb{Q}_{1}$ satisfy:

$$
\begin{gathered}
\operatorname{Re}\left(\frac{\zeta q^{\prime \prime}(\zeta)}{q^{\prime}(\zeta)}\right) \geq 0, \quad\left|\frac{\mathcal{H}_{\alpha, \beta}^{\eta+1, k}(f(z))-\mathcal{H}_{\alpha, \beta}^{\eta, k}(f(z))}{z q^{\prime}(\zeta)}\right| \leq\left|\frac{k}{b}\right| \ell, \\
\left\{\phi\left(\frac{\mathcal{H}_{\alpha, \beta}^{\eta, k}(f(z))}{z}, \frac{\mathcal{H}_{\alpha, \beta}^{\eta+1, k}(f(z))}{z}, \frac{\mathcal{H}_{\alpha, \beta}^{\eta+2, k}(f(z))}{z}, \frac{\mathcal{H}_{\alpha, \beta}^{\eta+3, k}(f(z))}{z} ; z\right): z \in \mathbb{U}\right\} \subset \Omega,
\end{gathered}
$$

then

$$
\mathcal{H}_{\alpha, \beta}^{\eta, k}(f(z)) \prec q(z)
$$

Proof. Let

$$
\mathcal{H}_{\alpha, \beta}^{\eta, k}(f(z))=z p(z) \quad z \in \mathbb{U},
$$

From (3), we have

$$
\frac{\mathcal{H}_{\alpha, \beta}^{\eta+1, k}(f(z))}{z}=\left(\frac{k}{b}\right)\left(z p^{\prime}(z)+\frac{b}{k} p(z)\right),
$$


which implies

$$
\frac{\mathcal{H}_{\alpha, \beta}^{\eta+2, k}(f(z))}{z}=\frac{k^{2}}{b(b+1)}\left(z^{2} p^{\prime \prime}(z)+\left(\frac{2 b+1}{k}+1\right) z p^{\prime}(z)+\frac{b(b+1)}{k^{2}} p(z)\right) .
$$

Furthermore, we have

$$
\begin{aligned}
\frac{\mathcal{H}_{\alpha, \beta}^{\eta+3, k}(f(z))}{z} & =\frac{k^{3}}{b(b+1)(b+2)}\left(z^{3} p^{\prime \prime \prime}(z)+3\left(\frac{b+1}{k}+1\right) z^{2} p^{\prime \prime}(z)\right. \\
& \left.+\left(\frac{3 b^{2}+6 b+2}{k^{2}}+\frac{3(b+1)}{k}+1\right) z p^{\prime}(z)+\frac{b(b+1)(b+2)}{k^{3}} p(z)\right) .
\end{aligned}
$$

Now, we define the parameters $a_{1}, a_{2}, a_{3}$, and $a_{4}$ as

$$
a_{1}=r, \quad a_{2}=\left(\frac{k}{b}\right)\left(s+\frac{b}{k} r\right), \quad a_{3}=\frac{k^{2}}{b(b+1)}\left(t+\left(\frac{2 b+1}{k}+1\right) s+\frac{b(b+1)}{k^{2}} r\right),
$$

and

$$
\begin{aligned}
a_{4}=\frac{k^{3}}{b(b+1)(b+2)}\left(u+3\left(\frac{b+1}{k}+1\right) t+\left(\frac{3 b^{2}+6 b+2}{k^{2}}\right.\right. & \left.+\frac{3(b+1)}{k}+1\right) s \\
& \left.+\frac{b(b+1)(b+2)}{k^{3}} r\right) .
\end{aligned}
$$

Then, transformation $\psi: \mathbb{C}^{4} \times \mathbb{U} \longrightarrow \mathbb{C}$ as

$$
\psi(r, s, t, u ; z)=\phi\left(a_{1}, a_{2}, a_{3}, a_{4} ; z\right),
$$

by using the relations from (9) to (12), we have

$$
\begin{aligned}
& \psi\left(p(z), z p^{\prime}(z), z^{2} p^{\prime \prime}(z), z^{3} p^{\prime \prime \prime}(z) ; z\right)= \\
& \phi\left(\frac{\mathcal{H}_{\alpha, \beta}^{\eta, k}(f(z))}{z}, \frac{\mathcal{H}_{\alpha, \beta}^{\eta+1, k}(f(z))}{z}, \frac{\mathcal{H}_{\alpha, \beta}^{\eta+2, k}(f(z))}{z}, \frac{\mathcal{H}_{\alpha, \beta}^{\eta+3, k}(f(z))}{z} ; z\right),
\end{aligned}
$$

therefore, we recompute (8) as

$$
\psi\left(p(z), z p^{\prime}(z), z^{2} p^{\prime \prime}(z), z^{3} p^{\prime \prime \prime}(z) ; z\right) \in \Omega,
$$

then, the proof is completed by showing that the admissibility condition for $\phi \in \Psi_{\Gamma}[\Omega, q(z)]$ is equivalent to the admissibility condition for $\psi$ as given in Definition 3, since

$$
\frac{t}{s}+1=\frac{(b+1)\left(a_{3}-a_{1}\right)}{k\left(a_{2}-a_{1}\right)}-\frac{2 b+1}{k}
$$

and

$$
\frac{u}{s}=\frac{(b+1)(b+2)\left(a_{4}-a_{1}\right)-3(b+1)(b+k+1)\left(a_{3}-a_{1}\right)}{k^{2}\left(a_{2}-a_{1}\right)}+\frac{3 b(b+1)+1}{k^{2}}+\frac{6 b+3}{k}+2,
$$

we also note that

$$
\left|\frac{z p^{\prime}(z)}{q^{\prime}(\zeta)}\right|=\left|\frac{\left(\frac{b}{z k}\right)\left(\mathcal{H}_{\alpha, \beta}^{\eta+1, k}(f(z))-\mathcal{H}_{\alpha, \beta}^{\eta, k}(f(z))\right)}{q^{\prime}(\zeta)}\right| \leq \ell,
$$


therefore, $\psi \in \Psi_{\Gamma}[\Omega, q(z)]$ and by Theorem $1, p(z) \prec q(z)$.

In a similar way, we define the parameters $a_{1}, a_{2}, a_{3}$, and $a_{4}$ as follows:

Definition 8. Let $\Omega \subseteq \mathbb{C}$ and $q(z) \in \mathbb{Q}$. The class of admissible functions $\Psi_{\Gamma}[\Omega, q(z)]$ consists of those functions $\phi: \mathbb{C}^{4} \times \mathbb{U} \longrightarrow \mathbb{C}$ that satisfy the admissibility condition

$$
\phi\left(a_{1}, a_{2}, a_{3}, a_{4} ; z\right) \notin \Omega,
$$

whenever

$$
\begin{gathered}
a_{1}=q(\zeta), \quad a_{2}=\frac{\ell \alpha \zeta q^{\prime}(\zeta)+c q(\zeta)}{c}, \\
\operatorname{Re}\left(\frac{(c-1)\left(a_{3}-a_{1}\right)}{\alpha\left(a_{2}-a_{1}\right)}-\frac{2 c-1}{\alpha}\right) \geq \ell \quad \operatorname{Re}\left(\frac{\zeta q^{\prime \prime}(\zeta)}{q^{\prime}(\zeta)}+1\right), \\
\operatorname{Re}\left(\frac{(c-1)(c-2)\left(a_{4}-a_{1}\right)-3(c-1)(c+\alpha-1)\left(a_{3}-a_{1}\right)}{\alpha^{2}\left(a_{2}-a_{1}\right)}+\frac{3 c(c-1)+1}{\alpha^{2}}\right. \\
\left.+\frac{6 c-3}{\alpha}+2\right) \geq \ell^{2} \operatorname{Re}\left(\frac{\zeta^{2} q^{\prime \prime \prime}(\zeta)}{q^{\prime}(\zeta)}\right),
\end{gathered}
$$

where $z \in \mathbb{U} ; \zeta \in \partial \mathbb{U} \backslash E(q), \ell \in \mathbb{N} \backslash\{1\}$ and $c=\alpha+\beta$.

Theorem 4. Let $\phi \in \Psi_{\Gamma}[\Omega, q(z)]$. If $f(z) \in \mathcal{H}$ and $q(z) \in \mathbb{Q}_{1}$ satisfy the following conditions:

$$
\begin{gathered}
\operatorname{Re}\left(\frac{\zeta q^{\prime \prime}(\zeta)}{q^{\prime}(\zeta)}\right) \geq 0, \quad\left|\frac{\frac{1}{z}\left[\mathcal{H}_{\alpha, \beta}^{\eta, k}(f(z))-\mathcal{H}_{\alpha, \beta+1}^{\eta, k}(f(z))\right]}{q^{\prime}(\zeta)}\right| \leq\left|\frac{\alpha}{c}\right| \ell, \\
\left\{\phi\left(\frac{\mathcal{H}_{\alpha, \beta+1}^{\eta, k}(f(z))}{z}, \frac{\mathcal{H}_{\alpha, \beta}^{\eta, k}(f(z))}{z}, \frac{\mathcal{H}_{\alpha, \beta-1}^{\eta, k}(f(z))}{z}, \frac{\mathcal{H}_{\alpha, \beta-2}^{\eta, k}(f(z))}{z} ; z\right): z \in \mathbb{U}\right\} \subset \Omega,
\end{gathered}
$$

then

$$
\mathcal{H}_{\alpha, \beta+1}^{\eta, k}(f(z)) \prec q(z) .
$$

Proof. Let

$$
\mathcal{H}_{\alpha, \beta+1}^{\eta, k}(f(z))=z p(z) \quad z \in \mathbb{U},
$$

From (4), we have

$$
\frac{\mathcal{H}_{\alpha, \beta}^{\eta, k}(f(z))}{z}=\left(\frac{\alpha}{c}\right)\left(z p^{\prime}(z)+\frac{c}{\alpha} p(z)\right),
$$

which implies

$$
\frac{\mathcal{H}_{\alpha, \beta-1}^{\eta, k}(f(z))}{z}=\frac{\alpha^{2}}{c(c-1)}\left(z^{2} p^{\prime \prime}(z)+\left(\frac{2 c-1}{\alpha}+1\right) z p^{\prime}(z)+\frac{c(c-1)}{\alpha^{2}} p(z)\right) .
$$

Moreover, we have

$$
\begin{aligned}
\frac{\mathcal{H}_{\alpha, \beta-2}^{\eta, k}(f(z))}{z} & =\frac{\alpha^{3}}{c(c-1)(c-2)}\left(z^{3} p^{\prime \prime \prime}(z)+3\left(\frac{c-1}{\alpha}+1\right) z^{2} p^{\prime \prime}(z)\right. \\
& \left.+\left(\frac{3 c^{2}-6 c+2}{\alpha^{2}}+\frac{3(c-1)}{\alpha}+1\right) z p^{\prime}(z)+\frac{c(c-1)(c-2)}{\alpha^{3}} p(z)\right) .
\end{aligned}
$$


Parameters $a_{1}, a_{2}, a_{3}$ and, $a_{4}$ as

$$
a_{1}=r, \quad a_{2}=\left(\frac{\alpha}{c}\right)\left(s+\frac{c}{\alpha} r\right), \quad a_{3}=\frac{\alpha^{2}}{c(c-1)}\left(t+\left(\frac{2 c-1}{\alpha}+1\right) s+\frac{c(c-1)}{\alpha^{2}} r\right)
$$

and

$$
\begin{aligned}
a_{4}=\frac{\alpha^{3}}{c(c-1)(c-2)}\left(u+3\left(\frac{c-1}{\alpha}+1\right) t+\left(\frac{3 c^{2}-6 c+2}{\alpha^{2}}+\right.\right. & \left.\frac{3(c-1)}{\alpha}+1\right) s \\
& \left.+\frac{c(c-1)(c-2)}{\alpha^{3}} r\right) .
\end{aligned}
$$

The transformation $\psi: \mathbb{C}^{4} \times \mathbb{U} \longrightarrow \mathbb{C}$

$$
\psi(r, s, t, u ; z)=\phi\left(a_{1}, a_{2}, a_{3}, a_{4} ; z\right),
$$

by using the relations from (17) to (20), we have

$$
\begin{aligned}
& \psi\left(p(z), z p^{\prime}(z), z^{2} p^{\prime \prime}(z), z^{3} p^{\prime \prime \prime}(z) ; z\right)= \\
& \phi\left(\frac{\mathcal{H}_{\alpha, \beta+1}^{\eta, k}(f(z))}{z}, \frac{\mathcal{H}_{\alpha, \beta}^{\eta, k}(f(z))}{z}, \frac{\mathcal{H}_{\alpha, \beta-1}^{\eta+2, k}(f(z))}{z}, \frac{\mathcal{H}_{\alpha, \beta-2}^{\eta, k}(f(z))}{z} ; z\right),
\end{aligned}
$$

we recompute (16) as

$$
\psi\left(p(z), z p^{\prime}(z), z^{2} p^{\prime \prime}(z), z^{3} p^{\prime \prime \prime}(z) ; z\right) \in \Omega,
$$

This completes the proof by showing that the admissibility condition for $\phi \in \Psi_{\Gamma}[\Omega, q(z)]$ is equivalent to the admissibility condition for $\psi$ as given in Definition 3, since

$$
\frac{t}{s}+1=\frac{(c-1)\left(a_{3}-a_{1}\right)}{\alpha\left(a_{2}-a_{1}\right)}-\frac{2 c-1}{\alpha},
$$

and

$$
\begin{aligned}
\frac{u}{s}=\frac{(c-1)(c-2)\left(a_{4}-a_{1}\right)-3(c-1)(c+\alpha-1)\left(a_{3}-a_{1}\right)}{\alpha^{2}\left(a_{2}-a_{1}\right)} & +\frac{3 c(c-1)+1}{\alpha^{2}}+\frac{6 c-3}{\alpha}+2,
\end{aligned}
$$

we also note that

$$
\left|\frac{z p^{\prime}(z)}{q^{\prime}(\zeta)}\right|=\left|\frac{\left(\frac{c}{z \alpha}\right)\left(\mathcal{H}_{\alpha, \beta}^{\eta, k}(f(z))-\mathcal{H}_{\alpha, \beta+1}^{\eta, k}(f(z))\right)}{q^{\prime}(\zeta)}\right| \leq \ell,
$$

therefore, $\psi \in \Psi_{\Gamma}[\Omega, q(z)]$ and hence by Theorem $1, p(z) \prec q(z)$.

If $\Omega \neq \mathbb{C}$ is simply connected to the domain, then $\Omega=h(\mathbb{U})$ for some conformal mapping $h(z)$ of $\mathbb{U}$ onto $\Omega$. In this case, the class $\Psi_{\Gamma}[h(\mathbb{U}), q(z)]$ is written as $\Psi_{\Gamma}[h, q]$; the following theorem is a direct consequence of Theorems 3 and 4 .

Theorem 5. Let $\phi \in \Psi_{\Gamma}[h, q]$. If $f(z) \in \mathcal{H}$ and $q(z) \in \mathbb{Q}_{1}$ satisfy the following conditions:

$$
\begin{gathered}
\text { (i) } \operatorname{Re}\left(\frac{\zeta q^{\prime \prime}(\zeta)}{q^{\prime}(\zeta)}\right) \geq 0, \quad\left|\frac{\frac{1}{z}\left[\mathcal{H}_{\alpha, \beta}^{\eta+1, k}(f(z))-\mathcal{H}_{\alpha, \beta}^{\eta, k}(f(z))\right]}{q^{\prime}(\zeta)}\right| \leq\left|\frac{k}{b}\right| \ell, \\
\text { (ii) } \phi\left(\frac{\mathcal{H}_{\alpha, \beta}^{\eta, k}(f(z))}{z}, \frac{\mathcal{H}_{\alpha, \beta}^{\eta+1, k}(f(z))}{z}, \frac{\mathcal{H}_{\alpha, \beta}^{\eta+2, k}(f(z))}{z}, \frac{\mathcal{H}_{\alpha, \beta}^{\eta+3, k}(f(z))}{z} ; z\right) \prec h(z),
\end{gathered}
$$


then

$$
\mathcal{H}_{\alpha, \beta}^{\eta, k}(f(z)) \prec q(z) .
$$

Theorem 6. Let $\phi \in \Psi_{\Gamma}[h, q]$. If $f(z) \in \mathcal{H}$ and $q(z) \in \mathbb{Q}_{1}$ satisfy the following conditions:

$$
\begin{gathered}
\operatorname{Re}\left(\frac{\zeta q^{\prime \prime}(\zeta)}{q^{\prime}(\zeta)}\right) \geq 0, \quad\left|\frac{\frac{1}{z}\left[\mathcal{H}_{\alpha, \beta}^{\eta, k}(f(z))-\mathcal{H}_{\alpha, \beta+1}^{\eta, k}(f(z))\right]}{q^{\prime}(\zeta)}\right| \leq\left|\frac{\alpha}{c}\right| \ell, \\
\phi\left(\frac{\mathcal{H}_{\alpha, \beta+1}^{\eta, k}(f(z))}{z}, \frac{\mathcal{H}_{\alpha, \beta}^{\eta, k}(f(z))}{z}, \frac{\mathcal{H}_{\alpha, \beta-1}^{\eta, k}(f(z))}{z}, \frac{\mathcal{H}_{\alpha, \beta-2}^{\eta, k}(f(z))}{z} ; z\right) \prec h(z),
\end{gathered}
$$

then

$$
\mathcal{H}_{\alpha, \beta+1}^{\eta, k}(f(z)) \prec q(z) .
$$

The next corollaries extend Theorems 3 and 4 , when the behavior of $q(z)$ on $\partial \mathbb{U}$ is not known.

Corollary 1. Let $\Omega \subset \mathbb{C}$ and let $q(z)$ be univalent in $\mathbb{U} ; q(0)=1$. Let $\phi \in \Psi_{\Gamma}\left[\Omega, q_{\sigma}\right]$ for some $\sigma \in(0,1)$ where $q_{\sigma}(z)=q(\sigma z)$. If $f(z) \in \mathcal{H}$ satisfies the following conditions:

$$
\begin{gathered}
\operatorname{Re}\left(\frac{\zeta q_{\sigma}^{\prime \prime}(\zeta)}{q_{\sigma}^{\prime}(\zeta)}\right) \geq 0, \quad\left|\frac{\frac{1}{z}\left[\mathcal{H}_{\alpha, \beta}^{\eta+1, k}(f(z))-\mathcal{H}_{\alpha, \beta}^{\eta, k}(f(z))\right]}{q_{\sigma}^{\prime}(\zeta)}\right| \leq\left|\frac{k}{b}\right| \ell, \\
\phi\left(\frac{\mathcal{H}_{\alpha, \beta}^{\eta, k}(f(z))}{z}, \frac{\mathcal{H}_{\alpha, \beta}^{\eta+1, k}(f(z))}{z}, \frac{\mathcal{H}_{\alpha, \beta}^{\eta+2, k}(f(z))}{z}, \frac{\mathcal{H}_{\alpha, \beta}^{\eta+3, k}(f(z))}{z} ; z\right) \in \Omega,
\end{gathered}
$$

then

$$
\mathcal{H}_{\alpha, \beta}^{\eta, k}(f(z)) \prec q(z)
$$

where $z \in \mathbb{U}$ and $\zeta \in \partial \mathbb{U} \backslash E\left(q_{\sigma}\right)$.

Proof. by using Theorem 3, we have $\mathcal{H}_{\alpha, \beta}^{\eta, k}(f(z)) \prec q_{\sigma}(z)$. Then we obtain the result from $q_{\sigma}(z) \prec q(z)$.

Corollary 2. Let $\Omega \subset \mathbb{C}$ and let $q(z)$ be univalent in $\mathbb{U} ; q(0)=1$. Let $\phi \in \Psi_{\Gamma}\left[\Omega, q_{\sigma}\right]$ for some $\sigma \in(0,1)$, where $q_{\sigma}(z)=q(\sigma z)$. If $f(z) \in \mathcal{H}$ satisfy the following conditions:

$$
\begin{gathered}
\operatorname{Re}\left(\frac{\zeta q_{\sigma}^{\prime \prime}(\zeta)}{q_{\sigma}^{\prime}(\zeta)}\right) \geq 0, \quad\left|\frac{\frac{1}{z}\left[\mathcal{H}_{\alpha, \beta}^{\eta, k}(f(z))-\mathcal{H}_{\alpha, \beta+1}^{\eta, k}(f(z))\right]}{q_{\sigma}^{\prime}(\zeta)}\right| \leq\left|\frac{\alpha}{c}\right| \ell, \\
\phi\left(\frac{\mathcal{H}_{\alpha, \beta+1}^{\eta, k}(f(z))}{z}, \frac{\mathcal{H}_{\alpha, \beta}^{\eta, k}(f(z))}{z}, \frac{\mathcal{H}_{\alpha, \beta-1}^{\eta, k}(f(z))}{z}, \frac{\mathcal{H}_{\alpha, \beta-2}^{\eta, k}(f(z))}{z} ; z\right) \in \Omega,
\end{gathered}
$$

then

$$
\mathcal{H}_{\alpha, \beta+1}^{\eta, k}(f(z)) \prec q(z),
$$

where $z \in \mathbb{U}$ and $\zeta \in \partial \mathbb{U} \backslash E\left(q_{\sigma}\right)$.

Proof. By using Theorem 4, we have $\mathcal{H}_{\alpha, \beta+1}^{\eta, k}(f(z)) \prec q_{\sigma}(z)$. Then we obtain the result from $q_{\sigma}(z) \prec q(z)$. 
Corollary 3. Let $\Omega \subset \mathbb{C}$ and let $q(z)$ be univalent in $\mathbb{U} ; q(0)=1$. Let $\phi \in \Psi_{\Gamma}\left[\Omega, q_{\sigma}\right]$ for some $\sigma \in(0,1)$, where $q_{\sigma}(z)=q(\sigma z)$. If $f(z) \in \mathcal{H}$ satisfy the following conditions:

$$
\begin{gathered}
\operatorname{Re}\left(\frac{\zeta q_{\sigma}^{\prime \prime}(\zeta)}{q_{\sigma}^{\prime}(\zeta)}\right) \geq 0, \quad\left|\frac{\frac{1}{z}\left[\mathcal{H}_{\alpha, \beta}^{\eta+1, k}(f(z))-\mathcal{H}_{\alpha, \beta}^{\eta, k}(f(z))\right]}{q_{\sigma}^{\prime}(\zeta)}\right| \leq\left|\frac{k}{b}\right| \ell, \\
\phi\left(\frac{\mathcal{H}_{\alpha, \beta}^{\eta, k}(f(z))}{z}, \frac{\mathcal{H}_{\alpha, \beta}^{\eta+1, k}(f(z))}{z}, \frac{\mathcal{H}_{\alpha, \beta}^{\eta+2, k}(f(z))}{z}, \frac{\mathcal{H}_{\alpha, \beta}^{\eta+3, k}(f(z))}{z} ; z\right) \prec h(z),
\end{gathered}
$$

then

$$
\mathcal{H}_{\alpha, \beta}^{\eta, k}(f(z)) \prec q(z),
$$

where $z \in \mathbb{U}$ and $\zeta \in \partial \mathbb{U} \backslash E\left(q_{\sigma}\right)$.

Corollary 4. Let $\Omega \subset \mathbb{C}$ and let $q(z)$ be univalent in $\mathbb{U} ; q(0)=1$. Let $\phi \in \Psi_{\Gamma}\left[\Omega, q_{\sigma}\right]$ for some $\sigma \in(0,1)$, where $q_{\sigma}(z)=q(\sigma z)$. If $f(z) \in \mathcal{H}$ satisfy the following conditions:

$$
\begin{gathered}
\operatorname{Re}\left(\frac{\zeta q_{\sigma}^{\prime \prime}(\zeta)}{q_{\sigma}^{\prime}(\zeta)}\right) \geq 0, \quad\left|\frac{\frac{1}{z}\left[\mathcal{H}_{\alpha, \beta}^{\eta, k}(f(z))-\mathcal{H}_{\alpha, \beta+1}^{\eta, k}(f(z))\right]}{q_{\sigma}^{\prime}(\zeta)}\right| \leq\left|\frac{\alpha}{c}\right| \ell, \\
\phi\left(\frac{\mathcal{H}_{\alpha, \beta+1}^{\eta, k}(f(z))}{z}, \frac{\mathcal{H}_{\alpha, \beta}^{\eta, k}(f(z))}{z}, \frac{\mathcal{H}_{\alpha, \beta-1}^{\eta, k}(f(z))}{z}, \frac{\mathcal{H}_{\alpha, \beta-2}^{\eta, k}(f(z))}{z} ; z\right) \prec h(z),
\end{gathered}
$$

then

$$
\mathcal{H}_{\alpha, \beta+1}^{\eta, k}(f(z)) \prec q(z),
$$

where $z \in \mathbb{U}$ and $\zeta \in \partial \mathbb{U} \backslash E\left(q_{\sigma}\right)$.

Theorem 7. Let $h(z)$ be univalent in $\mathbb{U}$. Let $\phi: \mathbb{C}^{4} \times \mathbb{U} \longrightarrow \mathbb{C}$. Suppose that the differential equation

$$
\begin{aligned}
\phi\left(q(z),\left(\frac{k}{b}\right)\left(z q^{\prime}(z)+\frac{b}{k} q(z)\right),\right. & \\
\frac{k^{2}}{b(b+1)}\left(z^{2} q^{\prime \prime}(z)+\left(\frac{2 b+1}{k}+1\right) z q^{\prime}(z)+\right. & \left.\frac{b(b+1)}{k^{2}} q(z)\right), \\
\frac{k^{3}}{b(b+1)(b+2)}\left(z^{3} q^{\prime \prime \prime}(z)+3\left(\frac{b+1}{k}+1\right) z^{2} q^{\prime \prime}(z)\right. & +\left(\frac{3 b^{2}+6 b+2}{k^{2}}+\frac{3(b+1)}{k}+1\right) z q^{\prime}(z) \\
& \left.\left.+\frac{b(b+1)(b+2)}{k^{3}} q(z)\right) ; z\right)=h(z),
\end{aligned}
$$

has a solution $q(z)$ with $q(0)=1$, which satisfies (7). If $f(z) \in \mathcal{H}$ satisfies (24) and

$$
\phi\left(\frac{\mathcal{H}_{\alpha, \beta}^{\eta, k}(f(z))}{z}, \frac{\mathcal{H}_{\alpha, \beta}^{\eta+1, k}(f(z))}{z}, \frac{\mathcal{H}_{\alpha, \beta}^{\eta+2, k}(f(z))}{z}, \frac{\mathcal{H}_{\alpha, \beta}^{\eta+3, k}(f(z))}{z} ; z\right),
$$

is analytic in $\mathbb{U}$, then

$$
\mathcal{H}_{\alpha, \beta}^{\eta, k}(f(z)) \prec q(z)
$$

and $q(z)$ is the best dominant of (27).

Proof. By using Theorem 3 that $q(z)$ is a dominant of (24). Since $q(z)$ satisfies (26), it is also a solution of (24) and therefore $q(z)$ will be dominated by all dominants. Hence, $q(z)$ is the best dominant.

Moreover, in a similar way, using Theorem 4, we have 
Theorem 8. Let $h(z)$ be univalent in $\mathbb{U}$. Let $\phi: \mathbb{C}^{4} \times \mathbb{U} \longrightarrow \mathbb{C}$. Suppose that the differential equation

$$
\begin{aligned}
\phi\left(q(z),\left(\frac{\alpha}{c}\right)\left(z q^{\prime}(z)+\frac{c}{\alpha} q(z)\right),\right. & \\
\frac{\alpha^{2}}{c(c-1)}\left(z^{2} q^{\prime \prime}(z)+\left(\frac{2 c-1}{\alpha}+1\right) z q^{\prime}(z)+\right. & \left.\frac{c(c-1)}{\alpha^{2}} q(z)\right), \\
\frac{\alpha^{3}}{c(c-1)(c-2)}\left(z^{3} q^{\prime \prime \prime}(z)+3\left(\frac{c-1}{\alpha}+1\right) z^{2} q^{\prime \prime}(z)\right. & +\left(\frac{3 c^{2}-6 c+2}{\alpha^{2}}+\frac{3(c-1)}{\alpha}+1\right) z q^{\prime}(z) \\
& \left.\left.+\frac{c(c-1)(c-2)}{\alpha^{3}} q(z)\right) ; z\right)=h(z),
\end{aligned}
$$

has a solution $q(z)$ with $q(0)=1$, which satisfies (15). If $f(z) \in \mathcal{H}$ satisfies (25) and

$$
\phi\left(\frac{\mathcal{H}_{\alpha, \beta+1}^{\eta, k}(f(z))}{z}, \frac{\mathcal{H}_{\alpha, \beta}^{\eta, k}(f(z))}{z}, \frac{\mathcal{H}_{\alpha, \beta-1}^{\eta, k}(f(z))}{z}, \frac{\mathcal{H}_{\alpha, \beta-2}^{\eta, k}(f(z))}{z} ; z\right),
$$

is analytic in $\mathbb{U}$, then

$$
\mathcal{H}_{\alpha, \beta+1}^{\eta, k}(f(z)) \prec q(z)
$$

and $q(z)$ is the best dominant of (29).

In the case $q(z)=1+M z,(M>0)$ and in view of the Definition 7 , the class of admissible functions $\Psi_{\Gamma}[\Omega, q]$ denoted by $\Psi_{\Gamma}[\Omega, M]$ is defined below.

Definition 9. Let $\Omega \subseteq \mathcal{C}$ and $M>0$. The class of admissible functions $\Psi_{\Gamma}[\Omega, M]$ consists of those functions $\phi: \mathbb{C}^{4} \times \mathcal{U} \longrightarrow \mathbb{C}$ that satisfy the admissibility condition

$$
\begin{gathered}
\phi\left(1+M e^{i \theta}, 1+\left(\frac{k}{b}\right)\left(\frac{b}{k}+\ell\right) M e^{i \theta},\right. \\
1+\frac{k^{2}}{b(b+1)}\left(L+\left(\frac{b(b+1)}{k^{2}}+\ell\left(\frac{2 b+1}{k}+1\right)\right) M e^{i \theta}\right), \\
1+\frac{k^{3}}{b(b+1)(b+2)}\left(N+3 L\left(\frac{b+1}{k}+1\right)+\left(\frac{b(b+1)(b+2)}{k^{3}}\right.\right. \\
\left.\left.\left.+\ell\left(\frac{3 b^{2}+6 b+2}{k^{2}}+\frac{3(b+1)}{k}+1\right)\right) M e^{i \theta}\right) ; z\right) \notin \Omega,
\end{gathered}
$$

where $z \in \mathbb{U}, \operatorname{Re}\left(L e^{-i \theta}\right) \geq \ell(\ell-1) M$ and $\operatorname{Re}\left(N e^{-i \theta}\right) \geq 0$ for all real $\theta$ and $\ell \in \mathbb{N} \backslash\{1\}$.

Corollary 5. Let $\phi \in \Psi_{\Gamma}[\Omega, M]$. If $f(z) \in \mathcal{H}$ satisfy the following conditions:

$$
\left|\frac{1}{z}\left[\mathcal{H}_{\alpha, \beta}^{\eta+1, k}(f(z))-\mathcal{H}_{\alpha, \beta}^{\eta, k}(f(z))\right]\right| \leq\left|\frac{k}{b}\right| \ell M,
$$

and

$$
\phi\left(\frac{\mathcal{H}_{\alpha, \beta}^{\eta, k}(f(z))}{z}, \frac{\mathcal{H}_{\alpha, \beta}^{\eta+1, k}(f(z))}{z}, \frac{\mathcal{H}_{\alpha, \beta}^{\eta+2, k}(f(z))}{z}, \frac{\mathcal{H}_{\alpha, \beta}^{\eta+3, k}(f(z))}{z} ; z\right) \in \Omega
$$

then

$$
\left|\frac{\mathcal{H}_{\alpha, \beta}^{\eta, k}(f(z))}{z}-1\right|<M
$$

Furthermore, with Definition 8, we can define the following: 
Definition 10. Let $\Omega \subseteq \mathcal{C}$ and $M>0$. The class of admissible functions $\Psi_{\Gamma}[\Omega, M]$ consists of those functions $\phi: \mathbb{C}^{4} \times \mathbb{U} \longrightarrow \mathbb{C}$ that satisfy the admissibility condition

$$
\begin{gathered}
\phi\left(1+M e^{i \theta}, 1+\left(\frac{\alpha}{c}\right)\left(\frac{c}{\alpha}+\ell\right) M e^{i \theta},\right. \\
1+\frac{\alpha^{2}}{c(c-1)}\left(L+\left(\frac{c(c-1)}{\alpha^{2}}+\ell\left(\frac{2 c-1}{\alpha}+1\right)\right) M e^{i \theta}\right), \\
1+\frac{\alpha^{3}}{c(c-1)(c-2)}\left(N+3 L\left(\frac{c-1}{\alpha}+1\right)+\left(\frac{c(c-1)(c-2)}{\alpha^{3}}\right.\right. \\
\left.\left.\left.+\ell\left(\frac{3 c^{2}-6 c+2}{\alpha^{2}}+\frac{3(c-1)}{\alpha}+1\right)\right) M e^{i \theta}\right) ; z\right) \notin \Omega,
\end{gathered}
$$

where $z \in \mathbb{U}, \operatorname{Re}\left(L e^{-i \theta}\right) \geq \ell(\ell-1) M$ and $\operatorname{Re}\left(N e^{-i \theta}\right) \geq 0$ for all real $\theta$ and $\ell \in \mathbb{N} \backslash\{1\}$.

Corollary 6. Let $\phi \in \Psi_{\Gamma}[\Omega, M]$. If $f(z) \in \mathcal{H}$ satisfy the following conditions:

$$
\left|\frac{1}{z}\left[\mathcal{H}_{\alpha, \beta}^{\eta, k}(f(z))-\mathcal{H}_{\alpha, \beta+1}^{\eta, k}(f(z))\right]\right| \leq\left|\frac{\alpha}{c}\right| \ell M
$$

and

$$
\phi\left(\frac{\mathcal{H}_{\alpha, \beta+1}^{\eta, k}(f(z))}{z}, \frac{\mathcal{H}_{\alpha, \beta}^{\eta, k}(f(z))}{z}, \frac{\mathcal{H}_{\alpha, \beta-1}^{\eta, k}(f(z))}{z}, \frac{\mathcal{H}_{\alpha, \beta-2}^{\eta, k}(f(z))}{z} ; z\right) \in \Omega
$$

then

$$
\left|\frac{\mathcal{H}_{\alpha, \beta+1}^{\eta, k}(f(z))}{z}-1\right|<M
$$

In the case $\Omega=q(\mathbb{U})=\{\omega:|\omega-1|<M,(M>0)\}$, we use notation $\Phi_{\Gamma}[M]$ to the class $\Phi_{\Gamma}[\Omega, M]$.

Corollary 7. Let $\phi \in \Psi_{\Gamma}[\Omega, M]$. If $f(z) \in \mathcal{H}$ satisfy the conditions (30) and

$$
\left|\phi\left(\frac{\mathcal{H}_{\alpha, \beta}^{\eta, k}(f(z))}{z}, \frac{\mathcal{H}_{\alpha, \beta}^{\eta+1, k}(f(z))}{z}, \frac{\mathcal{H}_{\alpha, \beta}^{\eta+2, k}(f(z))}{z}, \frac{\mathcal{H}_{\alpha, \beta}^{\eta+3, k}(f(z))}{z} ; z\right)-1\right|<M,
$$

then

$$
\left|\frac{\mathcal{H}_{\alpha, \beta}^{\eta, k}(f(z))}{z}-1\right|<M
$$

Putting $\phi\left(a_{1}, a_{2}, a_{3}, a_{4} ; z\right)=a_{2}=1+\left(\frac{k}{b}\right)\left(\frac{b}{k}+\ell\right) M e^{i \theta}$ in Corollary 7, we have the following corollary:

Corollary 8. Let $M>0$ and $b \in \mathbb{C} \backslash \mathbb{Z}_{0}^{-}$with $\operatorname{Re}(b)<\frac{-\ell}{2}, \ell \in \mathbb{N} \backslash\{1\}$. If $f(z) \in \mathcal{H}$ satisfy the condition (30), and also, if:

$$
\left|\frac{\mathcal{H}_{\alpha, \beta}^{\eta+1, k}(f(z))}{z}-1\right|<M
$$

then

$$
\left|\frac{\mathcal{H}_{\alpha, \beta}^{\eta, k}(f(z))}{z}-1\right|<M .
$$


Corollary 9. Let $\phi \in \Psi_{\Gamma}[\Omega, M]$. If $f(z) \in \mathcal{H}$ satisfy the conditions (31) and

$$
\left|\phi\left(\frac{\mathcal{H}_{\alpha, \beta+1}^{\eta, k}(f(z))}{z}, \frac{\mathcal{H}_{\alpha, \beta}^{\eta, k}(f(z))}{z}, \frac{\mathcal{H}_{\alpha, \beta-1}^{\eta, k}(f(z))}{z}, \frac{\mathcal{H}_{\alpha, \beta-2}^{\eta, k}(f(z))}{z} ; z\right)-1\right|<M,
$$

then

$$
\left|\frac{\mathcal{H}_{\alpha, \beta+1}^{\eta, k}(f(z))}{z}-1\right|<M
$$

Putting $\phi\left(a_{1}, a_{2}, a_{3}, a_{4} ; z\right)=a_{2}=1+\left(\frac{\alpha}{c}\right)\left(\frac{c}{\alpha}+\ell\right) M e^{i \theta}$ in Corollary 9, we have the following corollary:

Corollary 10. Let $M>0$ and $c \in \mathbb{C} \backslash \mathbb{Z}_{0}^{-}$with $\operatorname{Re}(c)<\frac{-\ell}{2}, \ell \in \mathbb{N} \backslash\{1\}$. If $f(z) \in \mathcal{H}$ satisfy the conditions (31) and

$$
\left|\frac{\mathcal{H}_{\alpha, \beta}^{\eta, k}(f(z))}{z}-1\right|<M
$$

then

$$
\left|\frac{\mathcal{H}_{\alpha, \beta+1}^{\eta, k}(f(z))}{z}-1\right|<M
$$

Corollary 11. Let $\ell \in \mathbb{N} \backslash 1, M>0$ and $b \in \mathbb{C} \backslash \mathbb{Z}_{0}^{-}$. If $f(z) \in \mathcal{H}$ satisfies the condition (30) and

$$
\left|\frac{1}{z}\left[\mathcal{H}_{\alpha, \beta}^{\eta+1, k}(f(z))-\mathcal{H}_{\alpha, \beta}^{\eta, k}(f(z))\right]\right| \leq\left|\frac{k}{b}\right| \ell M
$$

then

$$
\left|\frac{\mathcal{H}_{\alpha, \beta}^{\eta, k}(f(z))}{z}-1\right|<M
$$

Proof. Let $\phi\left(a_{1}, a_{2}, a_{3}, a_{4} ; z\right)=a_{2}-a_{1}$. Using Corollary 5 with $\Omega=h(\mathbb{U})$ and

$$
h(z)=\left|\frac{k}{b}\right| \ell M \quad(z \in \mathbb{U})
$$

Now we show that $\phi \in \Psi_{\Gamma}[\Omega, M]$. Since the condition (30) is satisfied from the condition (32) and

$$
\begin{gathered}
\mid \phi\left(1+M e^{i \theta}, 1+\left(\frac{k}{b}\right)\left(\frac{b}{k}+\ell\right) M e^{i \theta},\right. \\
1+\frac{k^{2}}{b(b+1)}\left(L+\left(\frac{b(b+1)}{k^{2}}+\ell\left(\frac{2 b+1}{k}+1\right)\right) M e^{i \theta}\right), \\
1+\frac{k^{3}}{b(b+1)(b+2)}\left(N+3 L\left(\frac{b+1}{k}+1\right)+\left(\frac{b(b+1)(b+2)}{k^{3}}\right.\right. \\
\left.\left.\left.+\ell\left(\frac{3 b^{2}+6 b+2}{k^{2}}+\frac{3(b+1)}{k}+1\right)\right) M e^{i \theta}\right) ; z\right)|=| \frac{k \ell M e^{i \theta}}{b}|=| \frac{k}{b} \mid \ell M,
\end{gathered}
$$

then we have the Corollary 11.

Corollary 12. Let $\ell \in \mathbb{N} \backslash\{1\}, M>0$ and $c \in \mathbb{C} \backslash \mathbb{Z}_{0}^{-}$. If $f(z) \in \mathcal{H}$ satisfies the condition (31) and

$$
\left|\frac{1}{z}\left[\mathcal{H}_{\alpha, \beta}^{\eta, k}(f(z))-\mathcal{H}_{\alpha, \beta+1}^{\eta, k}(f(z))\right]\right| \leq\left|\frac{\alpha}{c}\right| \ell M,
$$


then

$$
\left|\frac{\mathcal{H}_{\alpha, \beta+1}^{\eta, k}(f(z))}{z}-1\right|<M
$$

Proof. Let $\phi\left(a_{1}, a_{2}, a_{3}, a_{4} ; z\right)=a_{2}-a_{1}$. Using Corollary 6 with $\Omega=h(\mathbb{U})$ and

$$
h(z)=\left|\frac{\alpha}{c}\right| \ell M \quad(z \in \mathbb{U}) .
$$

Now we show that $\phi \in \Psi_{\Gamma}[\Omega, M]$. Since the condition (31) is satisfied from the condition (33) and

$$
\begin{aligned}
& \mid \phi\left(1+M e^{i \theta}, 1+\left(\frac{\alpha}{c}\right)\left(\frac{c}{\alpha}+\ell\right) M e^{i \theta},\right. \\
& 1+\frac{\alpha^{2}}{c(c-1)}\left(L+\left(\frac{c(c-1)}{\alpha^{2}}+\ell\left(\frac{2 c-1}{\alpha}+1\right)\right) M e^{i \theta}\right), \\
& 1+\frac{\alpha^{3}}{c(c-1)(c-2)}\left(N+3 L\left(\frac{c-1}{\alpha}+1\right)+\left(\frac{c(c-1)(c-2)}{\alpha^{3}}\right.\right. \\
& \left.\left.\left.\quad+\ell\left(\frac{3 c^{2}-6 c+2}{\alpha^{2}}+\frac{3(c-1)}{\alpha}+1\right)\right) M e^{i \theta}\right) ; z\right)|=| \frac{\alpha \ell M e^{i \theta}}{c}|=| \frac{\alpha}{c} \mid \ell M,
\end{aligned}
$$

then the corollary is completed.

Corollary 13. Let $\ell \in \mathbb{N} \backslash\{1\}, M>0$ and $b \in \mathbb{C} \backslash \mathbb{Z}_{0}^{-}$. If $f(z) \in \mathcal{H}$ satisfy the condition (30) and

$$
\begin{aligned}
\left|\frac{1}{z}\left[\mathcal{H}_{\alpha, \beta}^{\eta+3, k}(f(z))-\mathcal{H}_{\alpha, \beta}^{\eta+2, k}(f(z))\right]\right| & \leq 2\left|\frac{k^{3}}{b(b+1)(b+2)}\right| \\
& \left(\left|\frac{2 b+1}{k}+3\right|+\left|\frac{b(b+1)}{k^{2}}+\frac{2 b+1}{k}+1\right|\right) M,
\end{aligned}
$$

then

$$
\left|\frac{\mathcal{H}_{\alpha, \beta}^{\eta, k}(f(z))}{z}-1\right|<M .
$$

Proof. Let $\phi\left(a_{1}, a_{2}, a_{3}, a_{4} ; z\right)=a_{4}-a_{3}$. We use Corollary 5 with $\Omega=h(\mathbb{U})$

$$
h(z)=2\left|\frac{k^{3}}{b(b+1)(b+2)}\right|\left(\left|\frac{2 b+1}{k}+3\right|+\left|\frac{b(b+1)}{k^{2}}+\frac{2 b+1}{k}+1\right|\right) M z, \quad(z \in \mathbb{U}) .
$$


Now we show that $\phi \in \Psi_{\Gamma}[\Omega, M]$. Since

$$
\begin{aligned}
& \mid \phi\left(1+M e^{i \theta}, 1+\left(\frac{k}{b}\right)\left(\frac{b}{k}+\ell\right) M e^{i \theta},\right. \\
& 1+\frac{k^{2}}{b(b+1)}\left(L+\left(\frac{b(b+1)}{k^{2}}+\ell\left(\frac{2 b+1}{k}+1\right)\right) M e^{i \theta}\right), \\
& 1+\frac{k^{3}}{b(b+1)(b+2)}\left(N+3 L\left(\frac{b+1}{k}+1\right)+\left(\frac{b(b+1)(b+2)}{k^{3}}\right.\right. \\
& \left.\left.\left.+\ell\left(\frac{3 b^{2}+6 b+2}{k^{2}}+\frac{3(b+1)}{k}+1\right)\right) M e^{i \theta}\right) ; z\right) \mid \\
& =\left|\frac{k^{3}}{b(b+1)(b+2)}\left(N+\left(\frac{2 b+1}{k}+3\right) L+\ell\left(\frac{b(b+1)}{k^{2}}+\frac{2 b+1}{k}+1\right) M e^{i \theta}\right)\right| \\
& =\left|\frac{k^{3} e^{i \theta}}{b(b+1)(b+2)}\left(N e^{-i \theta}+\left(\frac{2 b+1}{k}+3\right) L e^{-i \theta}+\ell\left(\frac{b(b+1)}{k^{2}}+\frac{2 b+1}{k}+1\right) M\right)\right| \\
& \geq\left|\frac{k^{3}}{b(b+1)(b+2)}\right|\left(\operatorname{Re}\left(N e^{-i \theta}\right)+\left|\frac{2 b+1}{k}+3\right| \operatorname{Re}\left(L e^{-i \theta}\right)\right. \\
& \left.+\ell\left|\frac{b(b+1)}{k^{2}}+\frac{2 b+1}{k}+1\right| M\right) \\
& \geq\left|\frac{k^{3}}{b(b+1)(b+2)}\right|\left(\ell(\ell-1) M\left|\frac{2 b+1}{k}+3\right|+\ell\left|\frac{b(b+1)}{k^{2}}+\frac{2 b+1}{k}+1\right| M\right) \\
& \geq 2\left|\frac{k^{3}}{b(b+1)(b+2)}\right|\left(\left|\frac{2 b+1}{k}+3\right|+\left|\frac{b(b+1)}{k^{2}}+\frac{2 b+1}{k}+1\right|\right) M,
\end{aligned}
$$

we complete the proof of Corollary 13.

Corollary 14. Let $\ell \in \mathbb{N} \backslash\{1\}, M>0$ and $c \in \mathbb{C} \backslash \mathbb{Z}_{0}^{-}$. If $f(z) \in \mathcal{H}$ satisfy the condition (31) and

$$
\begin{aligned}
\left|\frac{1}{z}\left[\mathcal{H}_{\alpha, \beta-2}^{\eta, k}(f(z))-\mathcal{H}_{\alpha, \beta-1}^{\eta, k}(f(z))\right]\right| & \leq 2\left|\frac{\alpha^{3}}{c(c-1)(c-2)}\right| \\
& \left(\left|\frac{2 c-1}{\alpha}+3\right|+\left|\frac{c(c-1)}{\alpha^{2}}+\frac{2 c-1}{\alpha}+1\right|\right) M,
\end{aligned}
$$

then

$$
\left|\frac{\mathcal{H}_{\alpha, \beta+1}^{\eta, k}(f(z))}{z}-1\right|<M
$$

Proof. Let $\phi\left(a_{1}, a_{2}, a_{3}, a_{4} ; z\right)=a_{4}-a_{3}$. We use Corollary 6 with $\Omega=h(\mathbb{U})$

$$
h(z)=2\left|\frac{\alpha^{3}}{c(c-1)(c-2)}\right|\left(\left|\frac{2 c-1}{\alpha}+3\right|+\left|\frac{c(c-1)}{\alpha^{2}}+\frac{2 c-1}{\alpha}+1\right|\right) M z, \quad(z \in \mathbb{U}) .
$$


Now we show that $\phi \in \Psi_{\Gamma}[\Omega, M]$. Since

$$
\begin{aligned}
& \mid \phi\left(1+M e^{i \theta}, 1+\left(\frac{\alpha}{c}\right)\left(\frac{c}{\alpha}+\ell\right) M e^{i \theta},\right. \\
& 1+\frac{\alpha^{2}}{c(c-1)}\left(L+\left(\frac{c(c-1)}{\alpha^{2}}+\ell\left(\frac{2 c-1}{\alpha}+1\right)\right) M e^{i \theta}\right), \\
& 1+\frac{\alpha^{3}}{c(c-1)(c-2)}\left(N+3 L\left(\frac{c-1}{\alpha}+1\right)+\left(\frac{c(c-1)(c-2)}{\alpha^{3}}\right.\right. \\
& \left.\left.\left.+\ell\left(\frac{3 c^{2}-6 c+2}{\alpha^{2}}+\frac{3(c-1)}{\alpha}+1\right)\right) M e^{i \theta}\right) ; z\right) \mid \\
& =\left|\frac{\alpha^{3}}{c(c-1)(c-2)}\left(N+\left(\frac{2 c-1}{\alpha}+3\right) L+\ell\left(\frac{c(c-1)}{\alpha^{2}}+\frac{2 c-1}{\alpha}+1\right) M e^{i \theta}\right)\right| \\
& =\left|\frac{\alpha^{3} e^{-i \theta}}{c(c-1)(c-2)}\left(N e^{-i \theta}+\left(\frac{2 c-1}{\alpha}+3\right) L e^{-i \theta}+\ell\left(\frac{c(c-1)}{\alpha^{2}}+\frac{2 c-1}{\alpha}+1\right) M\right)\right| \\
& \geq\left|\frac{\alpha^{3}}{c(c-1)(c-2)}\right|\left(\operatorname{Re}\left(N e^{-i \theta}\right)+\left|\frac{2 c-1}{\alpha}+3\right| \operatorname{Re}\left(L e^{-i \theta}\right)\right. \\
& \left.+\ell\left|\frac{c(c-1)}{\alpha^{2}}+\frac{2 c-1}{\alpha}+1\right| M\right) \\
& \geq\left|\frac{\alpha^{3}}{c(c-1)(c-2)}\right|\left(\ell(\ell-1) M\left|\frac{2 c-1}{k}+3\right|+\ell\left|\frac{c(c-1)}{\alpha^{2}}+\frac{2 c-1}{\alpha}+1\right| M\right) \\
& \geq 2\left|\frac{\alpha^{3}}{c(c-1)(c-2)}\right|\left(\left|\frac{2 c-1}{\alpha}+3\right|+\left|\frac{c(c-1)}{\alpha^{2}}+\frac{2 c-1}{\alpha}+1\right|\right) M,
\end{aligned}
$$

we complete the proof of Corollary 14.

\section{Third Order Differential Supordination with $\mathcal{H}_{\alpha, \beta}^{\eta, k}(f(z))$}

Definition 11. Let $\Omega \subseteq \mathbb{C}$ and $q(z) \in \mathbb{Q}$. The class of admissible functions $\Psi_{\Gamma}[\Omega, q(z)]$ consists of those functions $\psi: \mathbb{C}^{4} \times \overline{\mathbb{U}} \longrightarrow \mathbb{C}$ that satisfy the admissibility condition

$$
\psi\left(a_{1}, a_{2}, a_{3}, a_{4} ; z\right) \notin \Omega,
$$

whenever

$$
\begin{gathered}
a_{1}=q(\zeta), \quad a_{2}=\frac{k \zeta q^{\prime}(\zeta)+b q(\zeta)}{b m}, \\
\operatorname{Re}\left(\frac{(b+1)\left(a_{3}-a_{1}\right)}{k\left(a_{2}-a_{1}\right)}-\frac{2 b+1}{k}\right) \leq \frac{1}{m} \quad \operatorname{Re}\left(\frac{\zeta q^{\prime \prime}(\zeta)}{q^{\prime}(\zeta)}+1\right), \\
\operatorname{Re}\left(\frac{(b+1)(b+2)\left(a_{4}-a_{1}\right)-3(b+1)(b+k+1)\left(a_{3}-a_{1}\right)}{k^{2}\left(a_{2}-a_{1}\right)}+\frac{3 b(b+1)+1}{k^{2}}\right. \\
\left.+\frac{6 b+3}{k}+2\right) \leq \frac{1}{m^{2}} \quad \operatorname{Re}\left(\frac{\zeta^{2} q^{\prime \prime \prime}(\zeta)}{q^{\prime}(\zeta)}\right),
\end{gathered}
$$

where $z \in \mathbb{U} ; \zeta \in \partial \mathbb{U}, m \in \mathbb{N} \backslash\{1\}$ and $b=\eta+k$. 
Theorem 9. Let $\phi \in \Psi_{\Gamma}^{\prime}[\Omega, q(z)]$. If $f(z) \in \mathcal{H}$ and $\frac{\mathcal{H}_{\alpha, \beta}^{\eta, k}(f(z))}{z} \in \mathbb{Q}_{1}$ satisfy the following conditions:

$$
\begin{aligned}
& \operatorname{Re}\left(\frac{z q^{\prime \prime}(z)}{q^{\prime}(z)}\right) \geq 0, \quad\left|\frac{\frac{1}{z}\left[\mathcal{H}_{\alpha, \beta}^{\eta+1, k}(f(z))-\mathcal{H}_{\alpha, \beta}^{\eta, k}(f(z))\right]}{q^{\prime}(z)}\right| \leq\left|\frac{k}{b}\right| m, \\
& \left\{\phi\left(\frac{\mathcal{H}_{\alpha, \beta}^{\eta, k}(f(z))}{z}, \frac{\mathcal{H}_{\alpha, \beta}^{\eta+1, k}(f(z))}{z}, \frac{\mathcal{H}_{\alpha, \beta}^{\eta+2, k}(f(z))}{z}, \frac{\mathcal{H}_{\alpha, \beta}^{\eta+3, k}(f(z))}{z} ; z\right): z \in \mathbb{U}\right\},
\end{aligned}
$$

are univalent, and

$$
\Omega \subset\left\{\phi\left(\frac{\mathcal{H}_{\alpha, \beta}^{\eta, k}(f(z))}{z}, \frac{\mathcal{H}_{\alpha, \beta}^{\eta+1, k}(f(z))}{z}, \frac{\mathcal{H}_{\alpha, \beta}^{\eta+2, k}(f(z))}{z}, \frac{\mathcal{H}_{\alpha, \beta}^{\eta+3, k}(f(z))}{z} ; z\right): z \in \mathbb{U}\right\},
$$

then

$$
q(z) \prec \frac{\mathcal{H}_{\alpha, \beta}^{\eta, k}(f(z))}{z} .
$$

Proof. Let the functions $p(z)$ and $\psi$ are defined by (9) and (13). Since $\phi \in \Psi_{\Gamma}^{\prime}[\Omega, q(z)]$, therefore (14) and (35) give

$$
\Omega \subset \psi\left(p(z), z p^{\prime}(z), z^{2} p^{\prime \prime}(z), z^{3} p^{\prime \prime \prime}(z) ; z\right) .
$$

The admissible condition for $\phi \in \Psi_{\Gamma}^{\prime}[\Omega, q(z)]$ is equivalent to the admissible condition for $\psi$ in Definition 6 with $n=2$. Therefore, $\psi \in \Psi_{\Gamma}^{\prime}[\Omega, q(z)]$ and by using (34) and Theorem 2, we have $q(z) \prec p(z)$ which yields $q(z) \prec \frac{\mathcal{H}_{\alpha, \beta}^{\eta, k}(f(z))}{z}$. Therefore, we complete the proof of Theorem 9.

Moreover, in a similar way, we can define the following:

Definition 12. Let $\Omega \subseteq \mathbb{C}$ and $q(z) \in \mathbb{Q}$. The class of admissible functions $\Psi_{\Gamma}[\Omega, q(z)]$ consists of those functions $\psi: \mathbb{C}^{4} \times \overline{\mathbb{U}} \longrightarrow \mathbb{C}$ that satisfy the admissibility condition:

$$
\psi\left(a_{1}, a_{2}, a_{3}, a_{4} ; z\right) \notin \Omega,
$$

whenever

$$
\begin{gathered}
a_{1}=q(\zeta), \quad a_{2}=\frac{\alpha \zeta q^{\prime}(\zeta)+c q(\zeta)}{c m} \\
\operatorname{Re}\left(\frac{(c-1)\left(a_{3}-a_{1}\right)}{\alpha\left(a_{2}-a_{1}\right)}-\frac{2 c-1}{\alpha}\right) \leq \frac{1}{m} \quad \operatorname{Re}\left(\frac{\zeta q^{\prime \prime}(\zeta)}{q^{\prime}(\zeta)}+1\right), \\
\operatorname{Re}\left(\frac{(c-1)(c-2)\left(a_{4}-a_{1}\right)-3(c-1)(c+\alpha-1)\left(a_{3}-a_{1}\right)}{\alpha^{2}\left(a_{2}-a_{1}\right)}+\frac{3 c(c-1)+1}{\alpha^{2}}\right. \\
\left.+\frac{6 c-3}{\alpha}+2\right) \leq \frac{1}{m^{2}} \quad \operatorname{Re}\left(\frac{\zeta^{2} q^{\prime \prime \prime}(\zeta)}{q^{\prime}(\zeta)}\right)
\end{gathered}
$$

where $z \in \mathbb{U} ; \zeta \in \partial \mathbb{U}, m \in \mathbb{N} \backslash\{1\}$ and $c=\alpha+\beta$.

With the assist of Definition 12 and Theorem 4, we have the following theorem 
Theorem 10. Let $\phi \in \Psi_{\Gamma}^{\prime}[\Omega, q(z)]$. If $f(z) \in \mathcal{H}$ and $\frac{\mathcal{H}_{\alpha, \beta+1}^{\eta, k}(f(z))}{z} \in \mathbb{Q}_{1}$ satisfy the following conditions:

$$
\begin{aligned}
& \operatorname{Re}\left(\frac{z q^{\prime \prime}(z)}{q^{\prime}(z)}\right) \geq 0, \quad\left|\frac{\frac{1}{z}\left[\mathcal{H}_{\alpha, \beta}^{\eta, k}(f(z))-\mathcal{H}_{\alpha, \beta+1}^{\eta, k}(f(z))\right]}{q^{\prime}(z)}\right| \leq\left|\frac{\alpha}{c}\right| m, \\
& \left\{\phi\left(\frac{\mathcal{H}_{\alpha, \beta+1}^{\eta, k}(f(z))}{z}, \frac{\mathcal{H}_{\alpha, \beta}^{\eta, k}(f(z))}{z}, \frac{\mathcal{H}_{\alpha, \beta-1}^{\eta, k}(f(z))}{z}, \frac{\mathcal{H}_{\alpha, \beta-2}^{\eta, k}(f(z))}{z} ; z\right): z \in \mathbb{U}\right\},
\end{aligned}
$$

are univalent, and

$$
\Omega \subset\left\{\phi\left(\frac{\mathcal{H}_{\alpha, \beta+1}^{\eta, k}(f(z))}{z}, \frac{\mathcal{H}_{\alpha, \beta}^{\eta, k}(f(z))}{z}, \frac{\mathcal{H}_{\alpha, \beta-1}^{\eta, k}(f(z))}{z}, \frac{\mathcal{H}_{\alpha, \beta-2}^{\eta, k}(f(z))}{z} ; z\right): z \in \mathbb{U}\right\},
$$

then

$$
q(z) \prec \frac{\mathcal{H}_{\alpha, \beta+1}^{\eta, k}(f(z))}{z} .
$$

If $\Omega \neq \mathbb{C}$ is a simply connected domain, then $\Omega=h(\mathbb{U})$ for some conformal mapping $h(z)$ of $\mathbb{U}$ onto $\Omega$. In this case, the class $\Psi_{\Gamma}^{\prime}[h(u), q(z)]$ is written as $\Psi_{\Gamma}^{\prime}[h, q]$. The following theorem is a direct consequence of Theorems 3 and 4 .

Theorem 11. Let $\phi \in \Psi_{\Gamma}^{\prime}[\Omega, q(z)]$ and $h(z)$ be analytic function in $\mathbb{U}$, and $f(z) \in \mathcal{H}$ and $\frac{\mathcal{H}_{\alpha, \beta}^{\eta, k}(f(z))}{z} \in \mathbb{Q}_{1}$ satisfy the condition (34). If

$$
\left\{\phi\left(\frac{\mathcal{H}_{\alpha, \beta}^{\eta, k}(f(z))}{z}, \frac{\mathcal{H}_{\alpha, \beta}^{\eta+1, k}(f(z))}{z}, \frac{\mathcal{H}_{\alpha, \beta}^{\eta+2, k}(f(z))}{z}, \frac{\mathcal{H}_{\alpha, \beta}^{\eta+3, k}(f(z))}{z} ; z\right): z \in \mathbb{U}\right\},
$$

is univalent function in $\mathbb{U}$, and

$$
h(z) \prec \phi\left(\frac{\mathcal{H}_{\alpha, \beta}^{\eta, k}(f(z))}{z}, \frac{\mathcal{H}_{\alpha, \beta}^{\eta+1, k}(f(z))}{z}, \frac{\mathcal{H}_{\alpha, \beta}^{\eta+2, k}(f(z))}{z}, \frac{\mathcal{H}_{\alpha, \beta}^{\eta+3, k}(f(z))}{z} ; z\right),
$$

then

$$
q(z) \prec \frac{\mathcal{H}_{\alpha, \beta}^{\eta, k}(f(z))}{z} .
$$

Theorem 12. Let $\phi \in \Psi_{\Gamma}^{\prime}[\Omega, q(z)]$ and $h(z)$ be analytic function in $\mathbb{U}$. If $f(z) \in \mathcal{H}$ and $\frac{\mathcal{H}_{\alpha, \beta+1}^{\eta, k}(f(z))}{z} \in \mathbb{Q}_{1}$ satisfy the condition (36). If

$$
\left\{\phi\left(\frac{\mathcal{H}_{\alpha, \beta+1}^{\eta, k}(f(z))}{z}, \frac{\mathcal{H}_{\alpha, \beta}^{\eta, k}(f(z))}{z}, \frac{\mathcal{H}_{\alpha, \beta-1}^{\eta+2, k}(f(z))}{z}, \frac{\mathcal{H}_{\alpha, \beta-2}^{\eta, k}(f(z))}{z} ; z\right): z \in \mathbb{U}\right\}
$$

is univalent function in $\mathbb{U}$ and

$$
h(z) \prec \phi\left(\frac{\mathcal{H}_{\alpha, \beta+1}^{\eta, k}(f(z))}{z}, \frac{\mathcal{H}_{\alpha, \beta}^{\eta, k}(f(z))}{z}, \frac{\mathcal{H}_{\alpha, \beta-1}^{\eta, k}(f(z))}{z}, \frac{\mathcal{H}_{\alpha, \beta-2}^{\eta, k}(f(z))}{z} ; z\right),
$$

then

$$
q(z) \prec \frac{\mathcal{H}_{\alpha, \beta+1}^{\eta, k}(f(z))}{z} .
$$


Theorem 13. Let $h(z)$ be analytic function in $\mathbb{U}$ and let $\psi: \mathbb{C}^{4} \times \mathbb{U} \longrightarrow \mathbb{C}$ and $\psi$ is given by (13). Suppose that the differential (26) has a solution $q(z) \in \mathbb{Q}_{1}$, and $f(z) \in \mathcal{H}$ satisfy the condition (34). If

$$
\left\{\phi\left(\frac{\mathcal{H}_{\alpha, \beta}^{\eta, k}(f(z))}{z}, \frac{\mathcal{H}_{\alpha, \beta}^{\eta+1, k}(f(z))}{z}, \frac{\mathcal{H}_{\alpha, \beta}^{\eta+2, k}(f(z))}{z}, \frac{\mathcal{H}_{\alpha, \beta}^{\eta+3, k}(f(z))}{z} ; z\right): z \in \mathbb{U}\right\},
$$

is univalent function in $\mathbb{U}$, and

$$
h(z) \prec \phi\left(\frac{\mathcal{H}_{\alpha, \beta}^{\eta, k}(f(z))}{z}, \frac{\mathcal{H}_{\alpha, \beta}^{\eta+1, k}(f(z))}{z}, \frac{\mathcal{H}_{\alpha, \beta}^{\eta+2, k}(f(z))}{z}, \frac{\mathcal{H}_{\alpha, \beta}^{\eta+3, k}(f(z))}{z} ; z\right),
$$

then

$$
q(z) \prec \frac{\mathcal{H}_{\alpha, \beta}^{\eta, k}(f(z))}{z} .
$$

and $q(z)$ is the best subordinant of relation (36).

Proof. The proof is similar to Theorem 7 and it is being omitted here.

Combining both Theorems 5 and 11, we have the following sandwich result:

Corollary 15. Let $h_{1}(z)$ and $q_{1}(z)$ be analytic functions in $\mathbb{U}$, also, let $h_{2}(z)$ be univalent in $\mathbb{U}, q_{2}(z) \in \mathbb{Q}_{1}$ with $q_{1}(0)=q_{2}(0)=1$ and $\phi \in \Psi_{\Gamma}[\Omega, q(z)] \cap \Psi_{\Gamma}^{\prime}[\Omega, q(z)]$. If $f(z) \in \mathcal{H}$ and $\frac{\mathcal{H}_{\alpha, \beta}^{\eta, k}(f(z))}{z} \in \mathbb{Q}_{1} \cap \mathcal{H}$,

$$
\left\{\phi\left(\frac{\mathcal{H}_{\alpha, \beta}^{\eta, k}(f(z))}{z}, \frac{\mathcal{H}_{\alpha, \beta}^{\eta+1, k}(f(z))}{z}, \frac{\mathcal{H}_{\alpha, \beta}^{\eta+2, k}(f(z))}{z}, \frac{\mathcal{H}_{\alpha, \beta}^{\eta+3, k}(f(z))}{z} ; z\right): z \in \mathbb{U}\right\},
$$

is univalent function in $\mathbb{U}$, and the conditions (22) and (34) are satisfied, also let

$$
h_{1}(z) \prec \phi\left(\frac{\mathcal{H}_{\alpha, \beta}^{\eta, k}(f(z))}{z}, \frac{\mathcal{H}_{\alpha, \beta}^{\eta+1, k}(f(z))}{z}, \frac{\mathcal{H}_{\alpha, \beta}^{\eta+2, k}(f(z))}{z}, \frac{\mathcal{H}_{\alpha, \beta}^{\eta+3, k}(f(z))}{z} ; z\right) \prec h_{2}(z),
$$

then

$$
q_{1}(z) \prec \frac{\mathcal{H}_{\alpha, \beta}^{\eta, k}(f(z))}{z} \prec q_{2}(z) .
$$

The proof of the following theorem is similar to Theorem 8 ; therefore, we omitted it.

Theorem 14. Let $h(z)$ be analytic function in $\mathbb{U}$ and let $\psi: \mathbb{C}^{4} \times \overline{\mathbb{U}} \longrightarrow \mathbb{C}$ and $\psi$ is given by (21). Suppose that the differential (28) has a solution $q(z) \in \mathbb{Q}_{1}$.If $f(z) \in \mathcal{H}$ satisfy the condition (36). If

$$
\left\{\phi\left(\frac{\mathcal{H}_{\alpha, \beta+1}^{\eta, k}(f(z))}{z}, \frac{\mathcal{H}_{\alpha, \beta}^{\eta, k}(f(z))}{z}, \frac{\mathcal{H}_{\alpha, \beta-1}^{\eta, k}(f(z))}{z}, \frac{\mathcal{H}_{\alpha, \beta-2}^{\eta, k}(f(z))}{z} ; z\right): z \in \mathbb{U}\right\},
$$

is univalent function in $\mathbb{U}$, and

$$
h(z) \prec \phi\left(\frac{\mathcal{H}_{\alpha, \beta+1}^{\eta, k}(f(z))}{z}, \frac{\mathcal{H}_{\alpha, \beta}^{\eta, k}(f(z))}{z}, \frac{\mathcal{H}_{\alpha, \beta-1}^{\eta, k}(f(z))}{z}, \frac{\mathcal{H}_{\alpha, \beta-2}^{\eta, k}(f(z))}{z} ; z\right),
$$

then

$$
q(z) \prec \frac{\mathcal{H}_{\alpha, \beta+1}^{\eta, k}(f(z))}{z} .
$$

and $q(z)$ is the best subordinant of (38). 
By combining Theorems 8 and 12, we obtain the following sandwich type result.

Corollary 16. Let $h_{1}(z)$ and $q_{1}(z)$ be analytic functions in $\mathbb{U}$ and leth $h_{2}(z)$ be univalent in $\mathbb{U}, q_{2}(z) \in \mathbb{Q}_{1}$ with $q_{1}(0)=q_{2}(0)=1$ and $\phi \in \Psi_{\Gamma}[\Omega, q(z)] \cap \Psi_{\Gamma}^{\prime}[\Omega, q(z)]$. If $f(z) \in \mathcal{H}$ and $\frac{\mathcal{H}_{\alpha, \beta+1}^{\eta, k}(f(z))}{z} \in \mathbb{Q}_{1} \cap \mathcal{H}$,

$$
\left\{\phi\left(\frac{\mathcal{H}_{\alpha, \beta+1}^{\eta, k}(f(z))}{z}, \frac{\mathcal{H}_{\alpha, \beta}^{\eta, k}(f(z))}{z}, \frac{\mathcal{H}_{\alpha, \beta-1}^{\eta, k}(f(z))}{z}, \frac{\mathcal{H}_{\alpha, \beta-2}^{\eta, k}(f(z))}{z} ; z\right): z \in \mathbb{U}\right\},
$$

is univalent function in $\mathbb{U}$, and the conditions (23) and (36) are satisfied; also let

$$
h_{1}(z) \prec \phi\left(\frac{\mathcal{H}_{\alpha, \beta+1}^{\eta, k}(f(z))}{z}, \frac{\mathcal{H}_{\alpha, \beta}^{\eta, k}(f(z))}{z}, \frac{\mathcal{H}_{\alpha, \beta-1}^{\eta, k}(f(z))}{z}, \frac{\mathcal{H}_{\alpha, \beta-2}^{\eta, k}(f(z))}{z} ; z\right) \prec h_{2}(z),
$$

then

$$
q_{1}(z) \prec \frac{\mathcal{H}_{\alpha, \beta+1}^{\eta, k}(f(z))}{z} \prec q_{2}(z) .
$$

\section{Conclusions}

By using the method of third-order differential subordination and superordination, we obtained many interesting results concerning the subordination and superordination properties of analytic functions associated with the operator $\mathcal{H}_{\alpha, \beta}^{\eta, k}(f)$.

Author Contributions: Data curation, M.F.Y.; Formal analysis, M.F.Y.; Funding acquisition, M.F.Y.; Investigation, A.A.A.; Methodology, A.A.A.; Project administration, P.A.; Resources, M.F.Y.; Writing-original draft, A.A.A.; Writing-review and editing, P.A. All authors have read and agreed to the published version of the manuscript.

Funding: This project was supported by the Deanship of Scientific Research at Prince Sattam Bin Abdulaziz University under the research project: 2019/01/10558.

Conflicts of Interest: The authors declare no conflict of interest.

\section{References}

1. Attiya, A.A. Some Applications of Mittag-Leffler Function in the Unit Disk. Filomat 2016, 30, $2075-2081$. [CrossRef]

2. Srivastava, H.M.; Tomovski, Z. Fractional calculus with an itegral operator containing a generalized Mittag-Leffler function in the kernal. Appl. Math. Comput. 2009, 211, 198-210.

3. Garg, M.; Manoha, P.; Kalla, S.L. A Mittag-Leffler-type function of two variables. Integral Transform. Spec. Funct. 2013, 24, 934-944. [CrossRef]

4. Kilbas, A.A.; Srivastava, H.M.; Trujillo, J.J. Theory and Applications of Fractional Differential Equations; North-Holland Mathematics Studies; Elsevier Science B.V.: Amsterdam, The Netherlands, 2006; Volume 204.

5. Kiryakova, V. Generalized Fractional Calculus and Applications; Pitman Research Notes in Mathematics Series; Longman Scientific \& Technical: Harlow, UK; John Wiley \& Sons Inc.: New York, NY, USA, 1994; Volume 301.

6. Kiryakova, V.S. Multiple, (multiindex) Mittag-Leffler functions and relations to generalized fractional calculus, Higher transcendental functions and their applications. J. Comput. Appl. Math. 2000, 118, 241-259. [CrossRef]

7. Kiryakova, V. The multi-index Mittag-Leffler functions as an important class of special functions of fractional calculus. Comput. Math. Appl. 2010, 59, 1885-1895. [CrossRef]

8. Mainardia, F.; Gorenflo, R. On Mittag-Leffler-type functions in fractional evolution processes, Higher transcendental functions and their applications. J. Comput. Appl. Math. 2000, 118, 283-299. [CrossRef]

9. Mittag-Leffler, G.M. Sur la nouvelle function. C. R. Acad. Sci. Paris 1903, 137, 554-558.

10. Mittag-Leffler, G.M. Sur la representation analytique d'une function monogene (cinquieme note). Acta Math. 1905, 29, 101-181. [CrossRef]

11. Ozarslan, M.A.; Yılmaz, B. The extended Mittag-Leffler function and its properties. J. Inequal. Appl. 2014, 2014, 85. [CrossRef] 
12. Podlubny, I. Fractional Differential Equations. An Introduction to Fractional Derivatives, Fractional Differential Equations, to Methods of Their Solution and Some of Their Applications. In Mathematics in Science and Engineering; Academic Press, Inc.: San Diego, CA, USA, 1999; Volume 198.

13. Prabhakar, T.R. A singular integral equation with a generalized Mittag-Leffler function in the Kernal. Yokohoma Math. J. 1971, 19, 7-15.

14. Prajapati, J.C.; Jana, R.K.; Saxena, R.K.; Shukla, A.K. Some results on the generalized Mittag-Leffler function operator. J. Inequal. Appl. 2013, 2013, 33. [CrossRef]

15. Shukla, A.K.; Prajapati, J.C. On a generalization of Mittag-Leffler function and its properties. J. Math. Anal. Appl. 2007, 336, 797-811. [CrossRef]

16. Tomovski, Z.; Hilfer, R.; Srivastava, H.M. Fractional and operational calculus with generalized fractional derivative operators and Mittag-Leffler type functions. Integral Transforms Spec. Funct. 2010, 21, 797-814. [CrossRef]

17. Tomovski, Z. Generalized Cauchy type problems for nonlinear fractional differential equations with composite fractional derivative operator. Nonlinear Anal. 2012, 75, 3364-3384. [CrossRef]

18. Wiman, A. Uber den Fundamental Salz in der Theorie der Funktionen. Acta. Math. 1905, 29, $191-201$. [CrossRef]

19. Yassen, M.F. Subordination results for certain class of analytic functions associated with Mittag-Leffler function. J. Comput. Anal. Appl. 2019, 26, 738-746.

20. Miller, S.S.; Mocanu, P.T. Subordinants of differential superordinations. Complex Var. Theory Appl. 2003, 48, 815-826. [CrossRef]

21. Tang, H.; Srivastava, H.M.; Li, S.H.; Ma, L.N. Third-order differential subordination and superordination results for meromorphically multivalent functions associated with the Liu-Srivastava operator. Abstr. Appl. Anal. 2014, 2014, 792175. [CrossRef]

22. Antonino, J.A.; Miller, S.S. Third-order differential inequalities and subordinations in the complex plane. Complex Var. Theory Appl. 2011, 56, 439-454. [CrossRef]

23. Attiya, A.A.; Kwon, O.S.; Hyang, P.J.; Cho, N.E. An integrodifferential operator for meromorphic functions associated with the Hurwitz-Lerch zeta function. Filomat 2016, 30, 2045-2057. [CrossRef]

Publisher's Note: MDPI stays neutral with regard to jurisdictional claims in published maps and institutional affiliations.

(C) 2020 by the authors. Licensee MDPI, Basel, Switzerland. This article is an open access article distributed under the terms and conditions of the Creative Commons Attribution (CC BY) license (http://creativecommons.org/licenses/by/4.0/). 